\title{
Desarrollo De Un Sistema Experto Para Mejorar La Asignacion Del Docente A Las Diferentes Catedras En La Facultad De Informática Y Electrónica Utilizando El Modelo De Mycin
}

\author{
Jonny Israel Guaiña Yungán \\ Byron Ernesto Vaca Barahona \\ Carlos Arturo Jara Santillán \\ Ángel Patricio Flores Orozco \\ Docente ESPOCH, Ecuador \\ Sttefano Israel Aguayo Cáceres
}

doi: 10.19044/esj.2017.v13n25p236 URL:http://dx.doi.org/10.19044/esj.2017.v13n25p236

\begin{abstract}
This research proposes the development of an expert system that improves the teaching assignment to teach the different chairs of the Faculty of Computer Science and Electronics at the Polytechnic School of Chimborazo.

The current problem involves a manual process of evaluation of academic profiles and find the best solution through an expert system that meets certain needs when assigning chairs to the teacher.

Free software was used. Linux Platform CentOS 5.0, Glassfish Server 3.1.2, PostgreSQL Database Version 9.2, Java Programming language with PrimeFaces 4.0 component library to automate, optimize and properly focus the assignment process.

Based on a Likert scale, the automated process was $93.33 \%$ efficient versus $33.33 \%$ of the manual process. Analyzing the results, it is concluded that the automatic process exceeds the manual process by $60 \%$, revealing that the process of assigning chairs has improved considerably using this expert system.

The use of this system will support and guide decisions when evaluating academic profiles of professionals who would like to be part of the faculty of the Faculty of Computer Science and Electronics at the Polytechnic Higher School of Chimborazo.
\end{abstract}

Keywords: Certainty Factors, Artificial Intelligence, (MYCIN) Expert Sysyem, Inference Engine, Evaluation System 


\section{Resumen}

La presente investigación propone el desarrollo de un sistema experto que permita asignar al mejor perfil académico de un docente para impartir asignaturas en una institución de educación superior.

La problemática actual, conlleva un proceso manual de evaluación de perfiles académicos y encontrar la mejor solución mediante un sistema experto que cubra ciertas necesidades a la hora de asignar cátedras al docente.

Se utilizó software de distribución libre. Plataforma Linux CentOS 5.0, Servidor Glassfish 3.1.2, Base de Datos PostgreSql versión 9.2, lenguaje de Programación Java con librería de componentes PrimeFaces 4.0 para automatizar, optimizar y enfocar adecuadamente el proceso de asignación de materias.

En base a una escala de Likert se obtuvo como resultado que el proceso automatizado tiene un $93.33 \%$ de eficiencia contra un $33.33 \%$ del proceso manual. Analizando los resultados sé concluye que el proceso automático supera en un $60.00 \%$ al proceso manual revelando que el proceso de asignación de cátedras ha mejorado considerablemente usando este sistema experto.

El uso de este sistema apoyara y guiara decisiones al momento de evaluar perfiles académicos de profesionales que quisieran formar parte de la institución de educación superior.

Palabras claves: Factores de Certeza, Inteligencia Artificial, Sistema Experto (MYCIN), Motor de Inferencia, Sistema de Evaluación

\section{Introdución}

Antes de la aparición del ordenador el hombre ya se preguntaba si le arrebatarían el privilegio de razonar, pensar y actuar. En la actualidad existe un campo dentro de la inteligencia artificial que desarrolla esta facultad: el de los sistemas expertos (SE). Estos sistemas también son conocidos como Sistemas basados en Conocimiento, según (Gochez, 2008) lo mencionado anteriormente permiten la creación de máquinas que razonan como el hombre mediante la utilización de programas, restringiéndose a un espacio de conocimientos limitados. En teoría pueden razonar siguiendo los pasos que sigue un experto humano (médico, analista, empresario) para resolver un problema concreto. (Gochez, 2008) menciona que este tipo de modelos de conocimiento por ordenador ofrece un extenso campo de posibilidades en resolución de problemas y en aprendizaje. Su uso será el extenderse ampliamente en el futuro, debido a su importante impacto sobre los negocios y la industria. 
(Inteligente, 2004) concluye que las características de los sistemas expertos permiten almacenar datos y conocimiento, para así sacar conclusiones lógicas, tomando decisiones que permiten aprender de la experiencia y los datos existentes explicando el porqué de las decisiones tomadas y realizar acciones como consecuencia de todo lo anterior.

Actualmente se puede notar casos donde docentes que tienen preparación académica en cierta área del saber están dictando materias de otra. Las causas para que esto suceda no es relevante en este estudio, sin embargo, este problema puede tener consecuencias en la calidad de estudio, afectando principalmente a los estudiantes, además según (Gochez, 2008) el proceso de selección de un perfil académico puede resultar bastante tedioso y tardado.

Todo cuanto un sistema experto se enfoca en resolver un problema específico sobre un área determinada, como los Sistemas: Tieresias (Herramienta para la transformación de conocimientos, 1972); Mycin (Diagnóstico de enfermedades de la sangre, 1972); Caduceus (Herramienta de diagnóstico para medicina interna, 1975); Prospector (Exploración mineral y herramientas de identificación, 1972); en el área de esta investigación existen Sistemas Informáticos que cubren parte de esta necesidad, la gran diferencia es que no son Sistemas Expertos.

En tal virtud se plantea el desarrollo de un Sistema Experto para mejorar la asignación de docentes a las diferentes cátedras, el mismo que sirva de apoyo a la toma de decisión en la parte Administrativa, además permitirá al personal docente ingresar datos académicos para que sean evaluado por el sistema y genere reportes con la respectiva información de compatibilidad de un perfil con ciertas materias.

El perfil profesional es un tema amplio, es por tanto aclarar que SADE está orientado al perfil académico que está inmerso dentro de un perfil profesional y que se puede validar con documentos legales, como es el caso de títulos de tercer nivel, cuarto nivel, cursos o certificaciones.

\section{Metodología}

Para realizar el presente trabajo se aplicó el método científico, porque se caracteriza por ser verificable, metódico, sistemático y capaz de elaborar predicciones en el campo de lo comprobable, además permite analizar y verificar las causas que generan el problema en el proceso de asignación docentes a las asignaturas. Para demostrar la incidencia del problema se realizará un levantamiento de la información mediante entrevistas al personal involucrado en este proceso para luego analizar, interpretar y difundir esos resultados.

Para el desarrollo del sistema informático se utilizó MYCIN: es un sistema experto que consta de dos elementos básicos: 1.- Base de 
Conocimientos y 2.- Mecanismo de Inferencia. La Base de Conocimientos está formada fundamentalmente por reglas del tipo "Si E, entonces H", representadas por E -> H, donde la premisa está en "forma conjuntiva" (es casi una cláusula de Horn) y la conclusión contiene un sólo predicado. El Mecanismo de Inferencia consiste en una estrategia de control de encadenamiento hacia atrás o dirigida por metas aplicada a las reglas. (Lope, 2005).

Reglas y hechos que se guarda en la base del conocimiento nos permitirá seleccionar el perfil más óptimo de un docente para el dictado de una determinada asignatura, el mismo que trabajará de la siguiente manera: Una vez conocida la información por parte de la máquina, el sistema experto plantea una hipótesis, para poder verificarlas, comprueba primero la exactitud de las premisas de las reglas. Esto se realiza mediante la búsqueda de enunciados correspondientes en la base de conocimientos, que pueden a su vez estar de nuevo en la parte de consultas de otra regla.

En el desarrollo de la Aplicación Web se utilizará la metodología Microsoft Solutions Framework (MSF). Que es una guía de desarrollo de software flexible que permite aplicar de manera individual e independiente cada uno de sus componentes, es escalable pues está diseñada para poder expandirse según la magnitud del proyecto. La metodología MSF está basada en un conjunto de principios, modelos, disciplinas, conceptos, directrices y practicas aprobadas por Microsoft, que asegura resultados con menor riesgo y de mayor calidad, centrándose en el proceso y las personas. Las fases que comprende esta metodología son: 1.- Visión, 2.- Planeación, 3.- Desarrollo, 4.- Estabilización, 5.- Implantación. (Pérez, 2011).

\section{Propuesta}

La presente investigación parte del desarrollo del Sistema de Asignación de Docentes ESPOCH (SADE), herramienta informática que es de apoyo a la toma de decisiones, para el proceso de contratación de docentes en la institución. SADE brinda una plataforma para que docentes registren información de su perfil académico que son evaluados por el sistema para posteriormente el personal administrativo verifiquen los resultados.

\section{Requerimientos funcioanles de sade}

Las razones que justifican la realización del Proyecto son:

- Mejorar el proceso de asignación de docentes a las materias a dictar.

- La satisfacción de estudiantes por tener docentes que sean expertos en los temas que dictan.

- Generación de reportes que permita a las autoridades tomar decisiones claves basadas en datos reales y exactos. 
Entre las funciones principales del sistema:

- $\quad$ Relleno de perfiles académicos de los docentes.

- Tomar decisiones con respecto a méritos académicos de los docentes.

- Visualización e impresión (impresiones físicas y digitales para apoyar al sector administrativo) de Reportes para la entrega y verificación de resultados en el debido proceso de contratación de docentes en la ESPOCH.

A continuación, se detalla el alcance de los requerimientos del sistema SADE donde se define los datos de entrada, procesos y salidas.

Implementación de seguridad a usuarios: El sistema tiene un módulo de seguridad donde cada usuario logre tener uno o más roles, a dichos roles se le otorga o niega permisos para las diferentes acciones del menú.

Registro de Nuevos Usuarios: El sistema permite el registro de nuevos usuarios aspirantes a docentes para acceder al sistema, es decir para los usuarios que no se encuentre en el sistema académico no tienen acceso directo al sistema sin un previo registro en el sistema SADE donde se otorga los permisos correspondientes.

Consumo de la estructura académica de la ESPOCH: El sistema permite el consumo de información de la estructura organizacional académica tales como: Facultades, Escuelas, Carreras y Materias.

Administración de procesos de contratación: El sistema permite al usuario administrar la creación de procesos, los mismos que contiene a los docentes que apliquen en dichos procesos.

Implementación del soporte necesario para los perfiles académicos: El sistema permite la gestión de información necesaria tales como títulos académicos, cursos y certificaciones que se otorgan a nivel nacional e internacional que sirvan de soporte para que los usuarios tengan la facilidad de registrar sus perfiles académicos.

Administración de la base de conocimiento: El sistema permite al personal autorizado administrar la base de conocimiento permitiendo agregar, modificar o eliminar reglas.

Administración del perfil académico de los aspirantes/docentes: El sistema permite al usuario aspirante/docente administrar la información de perfiles académicos ya sea para ingresar, modificar o eliminar sus títulos, cursos y certificaciones cuando el sistema lo permita.

Generación y consulta de reportes de aspirantes/docentes: El sistema permite la generación de reportes para el aspirante/docente una vez que haya seleccionado el paquete de materia para el cual desea aplicar, además dichos reportes se almacenan para posteriores consultas.

Consulta de reportes de directivos: El sistema permite hacer consultas a los directivos delegados del proceso de contratación, estas consultas pueden hacerse de dos maneras: En la primera opción el sistema le indica los 
paquetes de materias activos con los respectivos resultados de los docentes que hayan postulado y en la segunda opción se muestra el resultado de cada docente con el respectivo paquete de materias en el que haya postulado.

En el gráfico 1 se visualiza una vista Macro de lo que se ha mostrado en el bloque anterior es:

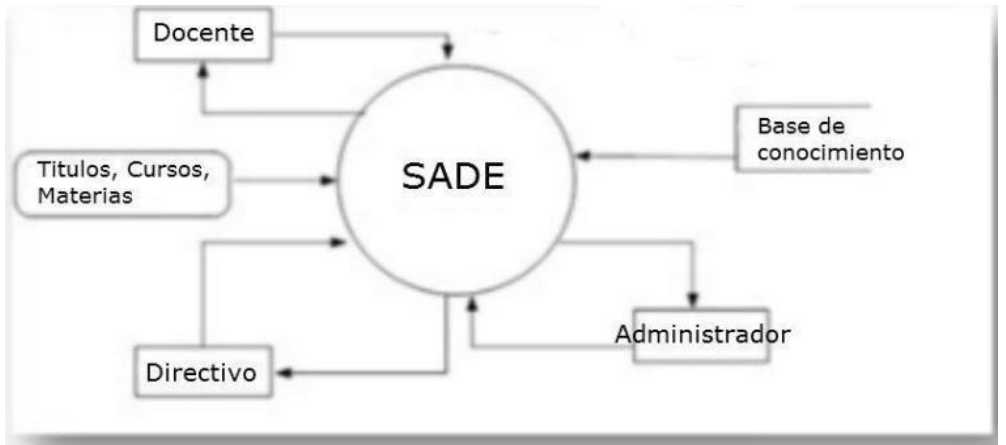

Gráfico 1. Arquitectura Externa

Autores: Denis Siavichay, Luis Parra

Nota: Las flechas representan el flujo de datos entre las principales entidades (y el proceso clases) Para mayor entendimiento el gráfico 2 muestra la misma arquitectura, pero a un nivel más alto:

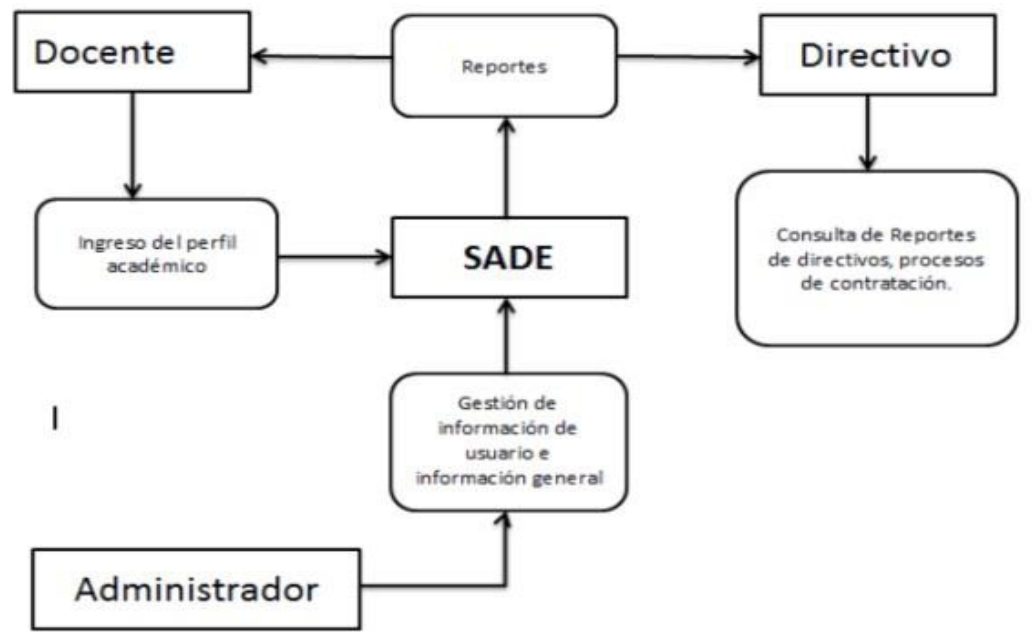

Gráfico 2. Arquitectura Detallada Autores: Denis Siavichay, Luis Parra

\section{Modelo Físico de la Base de Datos}

En el gráfico 3 se muestra el Modelo Físico de la Base de Datos con sus respectivas tablas, registros, tipos de variables y relaciones. 


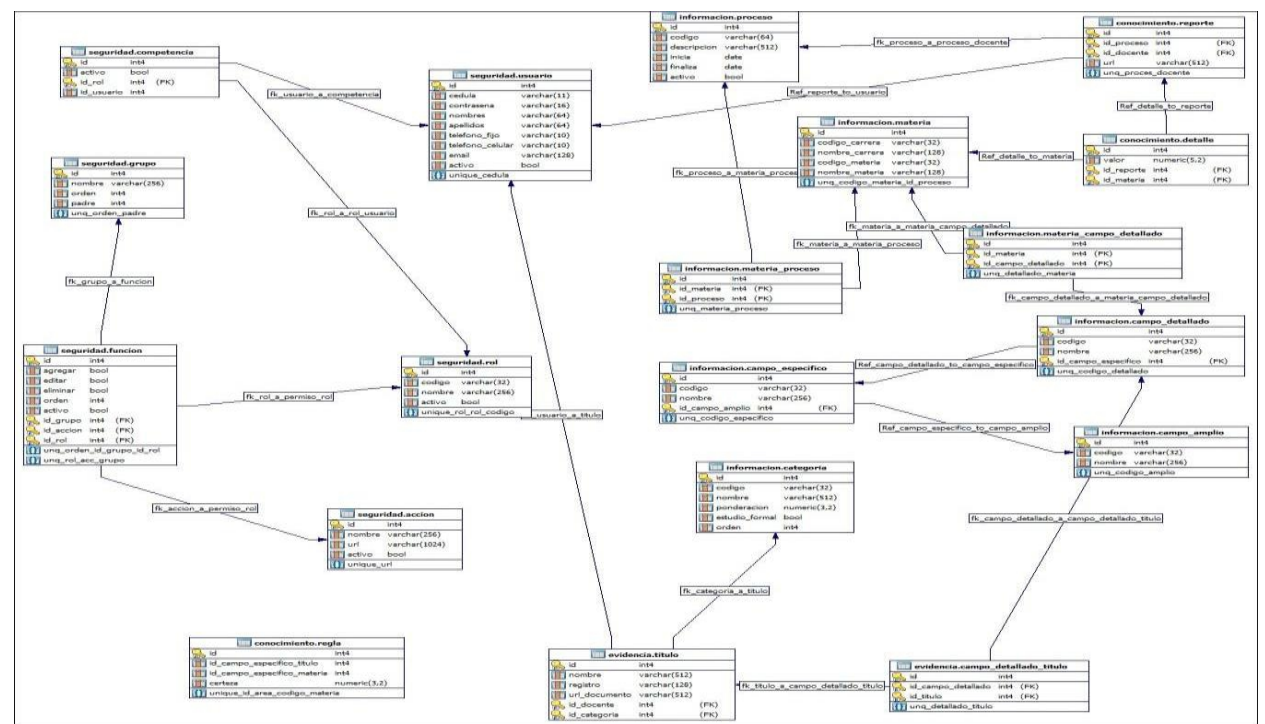

Gráfico 3. Modelo Físico BD

Autores: Denis Siavichay, Luis Parra

\section{Resultados y análisis}

En este punto se revela un análisis de los pasos que sigue manualmente para cumplir con un proceso de contratación frente al procedimiento automático que propone SADE.

Los pasos principales de este análisis son: Elaboración de la necesidad académica, Ingreso y postulación del perfil académico de un docente, Evaluación de los perfiles de los docentes y aspirantes y toma de decisión.

Para evaluar los pasos descritos anteriormente se ha recurrido a encuestas realizadas a docentes de la institución que reflejan tiempos de respuesta sobre el proceso manual de contratación, de la misma manera para el procedimiento automático que realiza sistema SADE se han ejecutado simulaciones con perfiles de docentes de la institución y paquetes de materia de la necesidad académica 2015, para determinar los tiempo de respuesta, cada uno de los resultados obtenidos se encuentran tabulados y representados en una escala de Likert.

\section{Tabulación de Datos del Proceso Manual y Proceso Automático Proceso Manual de Contratación}

En los siguientes gráficos de tabulaciones las opciones que se evalúan se encuentran en base a tiempos de respuesta los cuales representan:

$\mathrm{a}=$ Muy Malo $\mathrm{b}=$ Malo

$\mathrm{c}=$ regular

$\mathrm{d}=$ Bueno

e = Muy

Bueno 
La pregunta a evaluar es la siguiente: Encuesta Administrativo: ¿Cuál es el tiempo aproximado que se demora en elaborar la necesidad académica con el respectivo perfil del docente a contratar en un paquete de asignaturas? En el Gráfico 4 se observa la tabulación de los datos sobre el proceso de elaboración de la necesidad académica, mismo que indica que el $57.14 \%$ de votantes ha seleccionado la opción "a" por lo que se deduce que este procedimiento es muy Malo ya que se necesitan más de 8 horas para cumplirse.

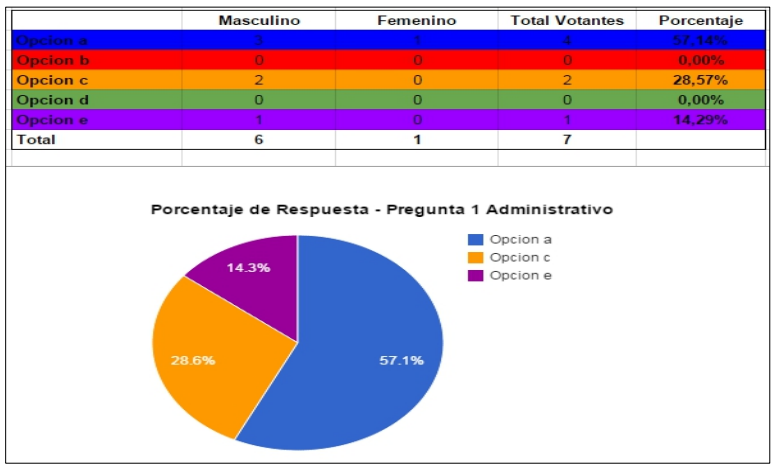

Autores: Denis Siavichay, Luis Parra

Gráfico 4. Tabulación de datos para Elaboración de la necesidad Académica

La siguiente pregunta a evaluar es Encuesta Docente: ¿Cuál es el tiempo que le toma cumplir con los requisitos habilitantes para entregar su carpeta académica en un concurso de méritos y oposición?

En el Gráfico 5 se observa la tabulación de los datos sobre el proceso de ingreso y postulación del perfil académico de un docente, mismo que indica que el $52.94 \%$ de votantes ha seleccionado la opción "a" por lo que se deduce que este procedimiento es muy malo ya que se necesitan más de 8 horas para cumplirse.

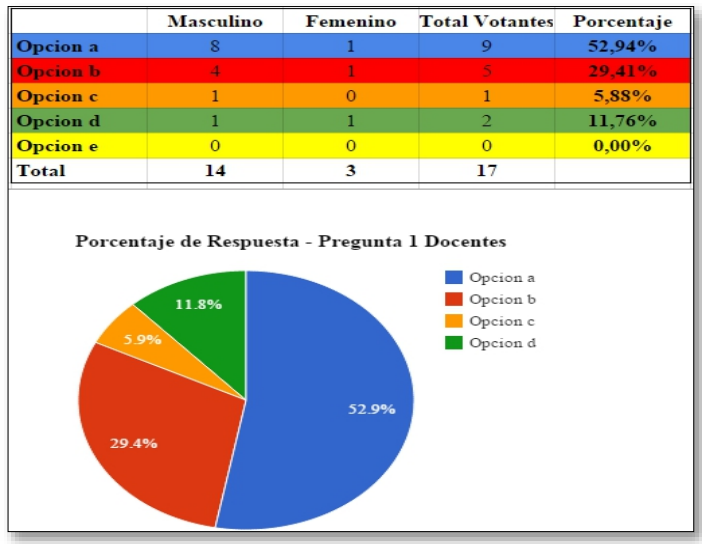

Gráfico 5. Tabulación de datos para ingreso y postulación del perfil académico de docente Autores: Denis Siavichay, Luis Parra 
La última pregunta a evaluar es Encuesta Administrativo: ¿Cuál es el tiempo necesario que le lleva la evaluación de la carpeta de un docente en el concurso de Méritos y Oposición?

En el Gráfico 6 se observa la tabulación de los datos sobre el proceso de evaluación y calificación del perfil de un docente, mismo que indica que el $42.86 \%$ de votantes ha seleccionado la opción "c" por lo que se deduce que este procedimiento es Regular ya que se necesitan de 2 a 4 horas para cumplirse.

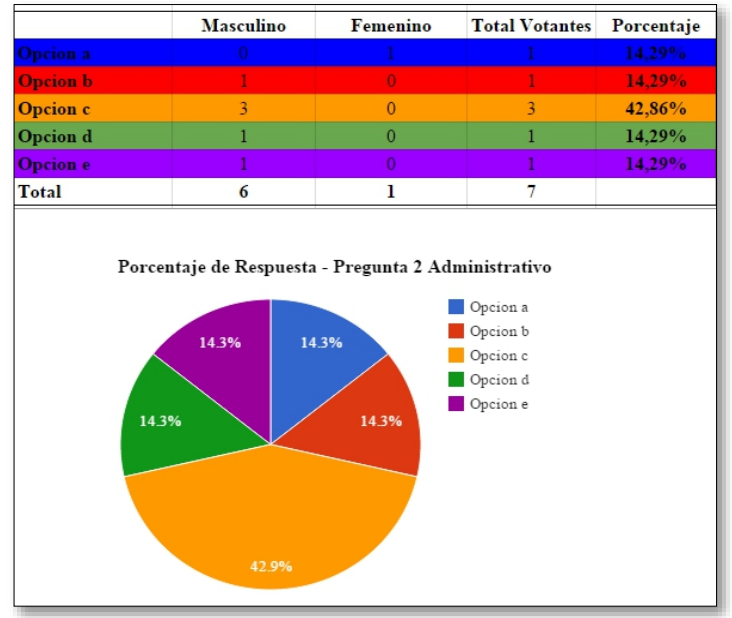

Autores: Denis Siavichay, Luis Parra

Gráfico 6. Tabulación de datos para evaluación de perfiles de docentes y aspirantes

Cuadro Comparativo del proceso manual vs proceso automatizado con SADE

\begin{tabular}{|c|c|c|c|c|c|c|c|c|c|}
\hline \multicolumn{5}{|c|}{ Manual } & \multicolumn{5}{|c|}{ Sistema SADE } \\
\hline \multicolumn{5}{|c|}{$\begin{array}{l}\text { El personal encargado elabora la } \\
\text { necesidad académica creando } \\
\text { paquetes de materias afines y } \\
\text { analizando los respectivos perfiles } \\
\text { necesarios para cubrir dicho paquete. }\end{array}$} & \multicolumn{5}{|c|}{$\begin{array}{l}\text { El personal encargado elabora la necesidad } \\
\text { académica creando paquetes de materias } \\
\text { a fines y los registran en el sistema. }\end{array}$} \\
\hline \multicolumn{5}{|c|}{$\begin{array}{l}\text { Tiempo Aproximado } \\
\text { más de } 8 \text { horas }\end{array}$} & \multicolumn{5}{|c|}{$\begin{array}{c}\text { Tiempo Aproximado } \\
1 \text { a } 2 \text { horas }\end{array}$} \\
\hline 1 & 2 & 3 & 4 & 5 & 1 & 2 & 3 & 4 & 5 \\
\hline $10 \%$ & $20 \%$ & $40 \%$ & $80 \%$ & $100 \%$ & $10 \%$ & $20 \%$ & $40 \%$ & $80 \%$ & $100 \%$ \\
\hline $\begin{array}{l}\mathbf{1}=\mathbf{M u} \\
\mathbf{2}=\mathbf{M} \mathbf{a} \\
\mathbf{3}=\mathbf{R e} \\
4=\mathbf{B u} \\
5=\mathbf{M u}\end{array}$ & $\begin{array}{l}\text { Malo } \\
\text { lo } \\
\text { ular } \\
\text { no } \\
\text { y buen }\end{array}$ & & & & $\begin{array}{l}1=\mathbf{M u} \\
2=\mathbf{M a} \\
3=\mathbf{R e g} \\
4=\mathbf{B u e} \\
5=\mathbf{M u}\end{array}$ & $\begin{array}{l}\text { Malo } \\
\text { o } \\
\text { ular } \\
\text { bo } \\
\text { bueno }\end{array}$ & & & \\
\hline
\end{tabular}


Elaboración de la necesidad académica

Tabla 6. Proceso 1

Autores: Denis Siavichay, Luis Parra

Proceso 1: Elaboración de lanecesidad académica

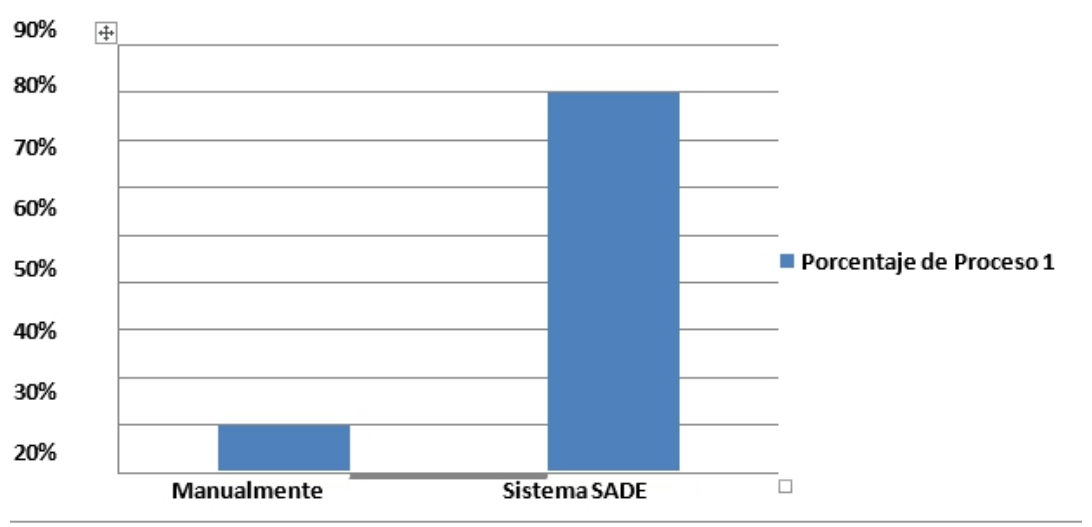

Gráfico 7. Proceso 1

Autores: Denis Siavichay, Luis Parra

Como se observa Tabla 6 y en el Grafico 7 el procedimiento de Elaboración de la necesidad académica se puede notar claramente los tiempos que se llega a requerir para cumplir con la necesidad tanto manualmente como automático. En el proceso Manual el personal encargado elabora la necesidad académica creando paquetes de materias afines y analizando los respectivos perfiles necesarios para cubrir dicho paquete, este este proceso se estima en un tiempo aproximado de más de 8 horas, mientras que en el proceso Automático el personal encargado elabora la necesidad académica creando paquetes de materias afines y los registran en el sistema sin necesidad de estudiar qué perfil académico es necesario ya que SADE se encarga de eso, todo este proceso toma aproximadamente de 1 a 2 horas. Al analizar ambos procesos se puede dar cuenta que el "Automático" es más eficiente que el proceso Manual el cual ayuda ahorrar tiempos de respuesta en un $70 \%$. 


\section{Evaluación de los perfiles de los docentes o aspirantes y toma de decisión}

Tabla 8. Proceso 3

\begin{tabular}{|c|c|}
\hline Manual & Sistema SADE \\
\hline $\begin{array}{c}\text { Después del plazo de recepción de } \\
\text { carpetas los encargados de la contratación } \\
\text { evalúan cada perfil para determinar los } \\
\text { mejores postulantes y concluir con la } \\
\text { nueva contratación. }\end{array}$ & $\begin{array}{c}\text { Los encargados de la contratación generan los } \\
\text { reportes ya sea por docente es decir el resultado } \\
\text { que tiene determinado docente o por paquete de } \\
\text { materias que muestra los mejores docentes para } \\
\text { cubrir un paquete, opcionalmente los directivos } \\
\text { pueden requerir la presentación de las carpetas de } \\
\text { los mejores aspirantes para constatar los datos } \\
\text { reflejados en el sistema y proceder con la toma de } \\
\text { decisión. }\end{array}$ \\
\hline
\end{tabular}

Autores: Denis Siavichay, Luis Parra

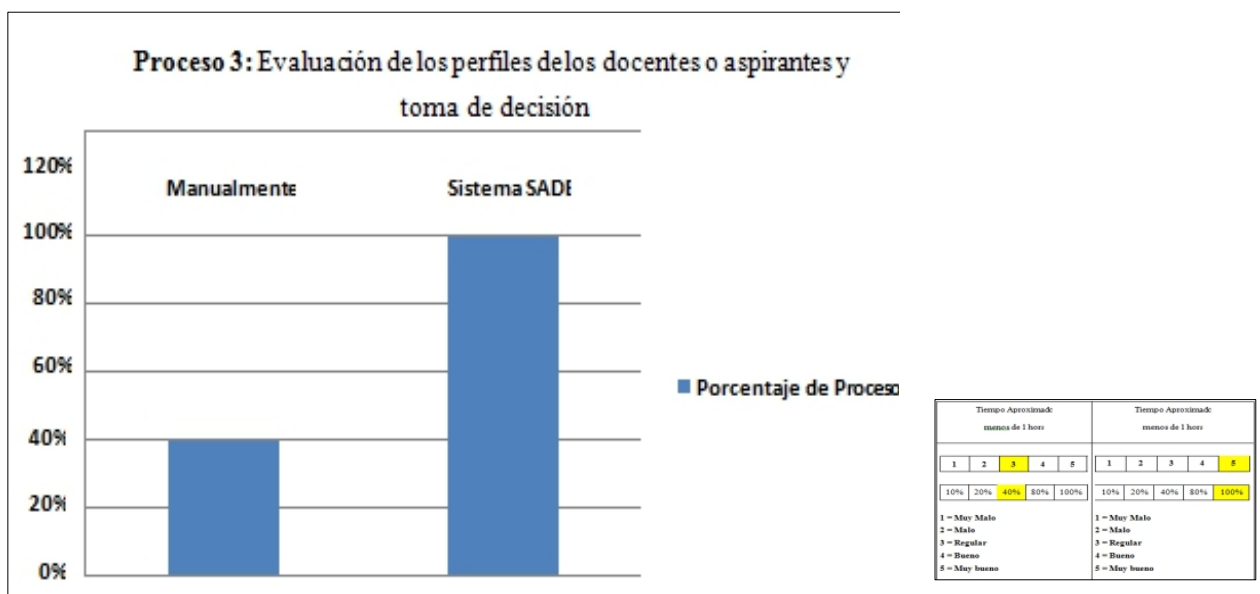

Gráfico 9. Proceso 3

Autores: Denis Siavichay, Luis Parra

Como se observa en la Tabla 8 y en el Gráfico 9 el Análisis de Perfiles de los Aspirantes es un proceso tedioso debido a que se debe revisar individualmente una pila de carpetas, este procedimiento puede tener una duración de 2 a 4 horas por perfil en el proceso manual, sin embargo, en el procedimiento automático es tan simple que los encargados de la contratación sólo deben pedirle al sistema que realice el análisis y este genera un reporte con los mejores perfiles, que toma también un tiempo menor a 1 hora. En base a lo dicho anteriormente se puede deducir que el proceso automático es más eficiente que el proceso manual en un $60 \%$. 


\section{Resultados}

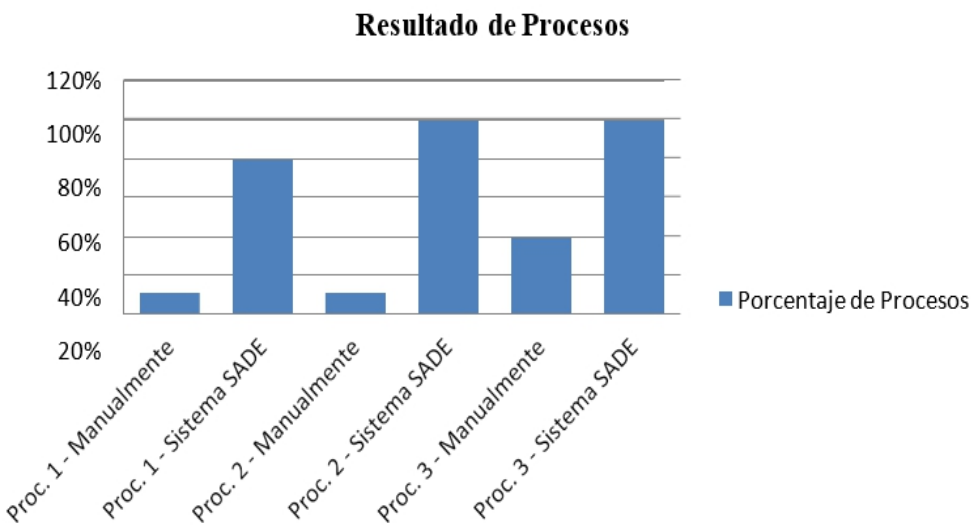

Gráfico 10. Resultados Estadísticos de los Procesos

Autores: Denis Siavichay, Luis Parra

Como se observa anteriormente en el Gráfico 10 en los tres procedimientos anteriores donde se compara los procesos manuales con los procesos Automáticos se puede notar claramente la diferencia en los tiempos que usa cada uno para cumplir estas necesidades. El primero procedimiento que trata sobre la "Elaboración de la necesidad académica" al comparar proceso manual y automático se deduce que el "Automático" es más eficaz que el proceso Manual con un $70 \%$ el cual ayuda ahorrar tiempos de respuesta. El segundo procedimiento que trata sobre el Ingreso o Postulación del perfil de un Docente que al comparar ambos procesos se puede deducir que el proceso automático es más eficiente que el proceso manual en un $90 \%$. Y por último el tercer procedimiento que trata sobre el Análisis de Perfiles de los Aspirantes se puede deducir que el proceso automático es más eficiente que el proceso manual en un $60 \%$.

\begin{tabular}{|c|c|c|c|}
\hline Proceso & Manual & Automático & Puntaje Ideal \\
\hline $\begin{array}{c}\text { Elaboración de la necesidad académica } \\
\text { Ingreso y postulación del perfil académico de un } \\
\text { docente }\end{array}$ & 1 & 4 & 5 \\
\hline $\begin{array}{c}\text { Evaluación de los perfiles de los docentes o } \\
\text { aspirantes y toma de decisión }\end{array}$ & 3 & 5 & 5 \\
\hline SUMA & 5 & 14 & 15 \\
\hline
\end{tabular}

Tabla 9 Resultados de los Procesos

Autores: Denis Siavichay, Luis Parra

Según la Tabla 9 se muestra los valores de cada uno de los procedimientos con sus respectivos procesos en base a la escala de Likert que igualmente se puede deducir que el sistema Automático es más eficiente en los procedimientos para evaluación de perfiles. Estos porcentajes y 
valores son conseguidos con pruebas y encuestas que se ha realizado a docentes de la Escuela Superior Politécnica de Chimborazo Extensión Morona Santiago. Después de analizar los 3 procedimiento se deduce 1o siguiente: $15=100 \%$ Puntaje ideal, $14=93.33 \%$ Proceso automático, $5=$ $33.33 \%$ Proceso Manual. Mediante estos resultados se puede concluir que el sistema Experto "SADE" es mucho más eficiente que el proceso manual en un $60,00 \%$ por lo tanto la Hipótesis queda comprobada ya que este sistema mejora la asignación de cátedras a los diferentes Docentes de la FIE.

\section{Conclusion}

Para realizar este trabajo de investigación se obtuvo las evidencias primitivas desde las mallas curriculares vigentes en la página web de la ESPOCH y los factores de certeza fueron conseguidas bajo conversaciones continuas con el personal administrativo de la ESPOCH Ext. Morona Santiago para así lograr el relleno de la Base de Conocimiento, para finalmente y por medio de los resultados descritos en la investigación se puede concluir que el sistema experto SADE mejora el proceso manual de evaluación de perfiles con una eficiencia superior en un $60.00 \%$.

Adicionalmente debemos mencionar que el sistema cumple satisfactoriamente con los requerimientos planteados en el desarrollo de software, debido a que la forma de acceder al sistema cumple con la propuesta plateada inicialmente.

\section{References:}

1. GOCHEZ, A. (28 de 01 de 2008). Inteligencia Artificial. Obtenido de http://inteligenciaartificialudb.blogspot.com/2008/01/concepto20caractersticas-y-metodologas.html

2. INTELIGENTE,I. I. (2004). III. Obtenido de http://www.informaticaintegral.net/sisexp.html

3. Pérez, O. A. (10 de junio de 2011). Cuatro enfoques metodológicos para el desarrollo de Software. Cuatro enfoques metodológicos para el desarrollo de Software.

4. LIBROS

5. MARTÍNEZ, Paloma. CASTRO, Elena, y otros., Diseño de Base de Datos - Problemas resueltos., Madrid - España., AlfaOmega Grupo Editor., 2001., 489 Pp.

6. ARAYA, Patricia., Manual curso básico de postgresql., San José Costa Rica., Autoedición., 2012., Pp.6 - 24.

7. LARMAN, Craig., Applying UML and Patterns: An Introduction to Object-Oriented Analysis and Design and the Unified Process., 3a ed., New Jersey - Estados Unidos., Prentice Hall PTR., 2004., Pp.6,10,13-16,22,28,29. 
8. HARMON, Paul. KING, David., Sistemas Expertos., Madrid España., Ediciones Díaz de Santos S.A., 1988., Pp.5,19-28,47-68.

9. E-BOOKS

10. MOLDES, Francisco., Java 7., Madrid-España., Anaya Multimedia Editorial., 2011., 416 Pp. http://www.amazon.es/Java-ManualesImprescindibles-Javier- Moldes/dp/8441529876.

11. VARAKSIM, Oleg. CALISKAN, Mert., Primefaces CookBook., Birmingham - Inglaterra., Packt Publishing., 2013., 328 Pp. http://download2.polytechnic.edu.na/pub4/sourceforge/d/do/docdoc/p fcb.pdf.

12. NORMAS TÉCNICAS

13. FRANCIA UNESCO., Revisión de la clasificación internacional normalizada de la educación: campos de la educación y la formación (CINE-F)., 37ª reunión., Paris - Francia., 2013., Pp 17 -19.

14. TESIS

15. LEÓN, Tomás., Sistemas Expertos y sus aplicaciones. Pachuca de soto - Mexico., Instituto de Ciencias Básicas e Ingenieria, Universidad Autónoma del Estado de Hidalgo., Tesis., 2007., Pp 151, 48-5.

16. INTERNET

17. PROGRAMACIÓN ORIENTA A OBJETOS

18. URL:

http://www.ecured.cu/index.php/Polimorfismo_(Inform\%C3\%A1tica ) 2012-09-05

19. PROGRAMACIÓN WEB

20. URL:

http://www.programacion.com/articulo/introduccion_a_la_tecnologia _javaserve r_faces_2332012-09-06

21. URL:http://www.adictosaltrabajo.com/tutoriales/tutoriales.php?pagin a=IntroduccionJ SFJava2012-09-07

22. URL:http://www.buenastareas.com/ensayos/EnfoqueEst\%C3\%A1tico-y- Din\%C3\%A1mico/5029729.html2012-09-11

23. URL: http://kalistog.wordpress.com/javaserver-faces-jsf 2012-09.12

24. URL: http://www.infosoftw.com/JSFNetBeansTutorialxHTML/ (Tutorial de JSF con NetBeans)2012-09-13

25. URL:http://ccia.ei.uvigo.es/docencia/SCS/1011/transparencias/Tema 5- 3.JSF.pdf2012-09-13

26. URL: http://www.htmlquick.com/es/reference/tags.html 2012-09-17

27. ARQUITECTURA MODELO-VISTA-CONTROLADOR

28. URL:http://www.nosolounix.com/2010/03/frameworks-modelovista- controlador-mvc.html2012-09-14

29. URL: http://www.tufuncion.com/mvc 2012-09-14 
30. SERVIDOR WEB

31. URL: http://www.adslfaqs.com.ar/glassfish-21-b60-servidor-paraaplicaciones- java-ee2012-09-18

32. INTELIGENCIA ARTIFICIAL

33. URL:http://inteligenciaartificialudb.blogspot.com/2008/01/conceptocaractersticasy-metodologas.html2012-09-19

34. SISTEMAS EXPERTOS 